\title{
Evidence unclear on whether Type I or II diabetes increases the risk of implant failure
}

\author{
Abstracted from \\ Chrcanovic BR, Albrektsson T, Wennerberg A. \\ Diabetes and oral implant failure: a systematic review. J Dent Res 2014; 93: 859-867. \\ Address for correspondence: B. Chrcanovic, Department of Prosthodontics, \\ Faculty of Odontology, Malmö University, Malmö, Sweden. E-mail: bruno.chrcanovic@mah.se
}

\section{Question: What is the influence of diabetes on dental implant failure rates}

Data sources PubMed, Web of Science and the Cochrane Oral Health Group Trials Register, www.clinicaltrials.gov, www.centerwatch.com and www.clinicalconnection.com databases. Manual searches of a number of dental journals and the reference lists of identified studies were undertaken.

Study selection Human clinical studies comparing implant failure rates in diabetic and non-diabetic patients were considered. Three reviewers independently selected studies.

Data extraction and synthesis The definition of implant failure used was complete loss of the implant. Study quality was assessed using the Cochrane risk of bias approach. A narrative summary of the studies and meta-analysis are presented.

Results Fourteen studies were included (one randomised controlled trial, six controlled clinical trials and seven retrospective analysis); all 14 were considered to be at high risk of bias. Meta-analysis (14 studies) found no significant difference between diabetic and nondiabetic patients; risk ratio of $1.07(95 \% \mathrm{Cl}=0.80,1.44)(\mathrm{p}=.65)$. A meta-analysis of two studies found a statistically significant difference (mean difference $=0.20,95 \% \mathrm{Cl}=0.08,0.31 \mathrm{p}=.001$;) between diabetic and non-diabetic patients concerning marginal bone loss, favouring non-diabetic patients. Meta-analysis was not possible for postoperative infections.

Conclusions The results of the present systematic review should be interpreted with caution because of the presence of uncontrolled confounding factors in the included studies. Within the limits of the existing investigations, the difference between the insertion of dental implants in non-diabetic and diabetic patients did not statistically affect the implant failure rates.

\section{Commentary}

This review asked an apparently clear question: what is the impact of diabetes on the success rate of implants? However, as the authors themselves discuss in a very balanced way, 'diabetes' is not a single condition and nor does it affect different individuals in the same way. Apart from there being Type I and II diabetic conditions, which may or may not in themselves affect metabolic control, glycaemic control is also thought to affect wound healing and varies between and within individual diabetics.

I think these authors could have attempted to answer at least two different sub-questions, which may have allowed clinicians to understand the potential complications in patients better:

1. What is the difference in implant success between

'well-controlled' Type I, Type II diabetics and non-diabetics?

2. What is the difference in implant success for diabetics with

different levels of glycaemic control compared to non-diabetics?

Alas, these weren't asked explicitly though the authors did seek to interpret and explain the included studies with these thoughts in mind. They were hampered in their ability to answer them by the quality of the primary data.

The authors conducted a thorough search, and study selection and data extraction methods were well done. I doubt whether any significant studies were not identified or data from studies missed.

Studies were eligible if they were 'clinical human studies comparing implant failure rates in diabetic and [non-diabetic] patients'. As it is impossible in a humane society to allocate patients to be diabetic or non-diabetic (randomly or otherwise), we can only observe the difference in success rates when implants are placed in these two groups.

Well-designed prospective cohort studies offer the best way to control for the biases that randomisation would otherwise overcome. In order to assess the risk that diabetes (Type I or Type II, controlled or not controlled) leads to an increase in implant failure we would want to control all other variables that could potentially do this. Thus the non-diabetic group should be matched so that there are the same number of smokers, similar age distribution, similar comorbidities, teeth affected by periodontitis etc as the diabetic groups, or both groups restricted to avoid potential confounders. Additionally, post-study controlling using statistical means can help to minimise the potential biases from confounding variables.

I mention this because the authors classify six studies as 'controlled clinical trials'. The Cochrane Handbook describes a study as being a trial when 'individuals (or other units)...were definitely or possibly 
allocated prospectively to one of two...alternative forms of health care...' ${ }^{1}$ Having diabetes or not cannot be allocated and is also not a health care intervention so I would argue that these studies cannot - regarding the variable of interest - be described as clinical trials but should instead be described as prospective cohort studies. We can have two arms of a trial that compares two different implant techniques and, from the one arm extract the data for diabetics and non-diabetics, but this is still a cohort study albeit conducted within a trial.

In addition, the authors classified seven of the 14 studies as 'retrospective analyses'. The two main retrospective study types we use in clinical epidemiology are retrospective cohort studies and case-control studies. It is unclear which, or whether both, were eligible, though my hunch is that it was cohort studies.

It may be that by classifying some of these observational studies as controlled clinical trials led the authors to then assess their quality using the Cochrane Risk of Bias tool. This tool was developed for trials rather than observational studies and as such any study that doesn't include randomisation or blinding is immediately considered at high risk of bias. But in observational studies we do not randomise and, at least for the participants, blinding is usually not possible. More important is that steps are taken to control for the confounders, something the Cochrane tool doesn't address. Thus it would have been more appropriate to assess their risk of bias using a tool designed specifically for observational studies, several of which exist, and for which the following domains are considered critical: ${ }^{2}$

- Methods for selecting study participants

- Methods for measuring exposure and outcome variables

- Design-specific sources of bias (excluding confounding)

- Methods to control confounding

- Statistical methods (excluding control of confounding)

Without going back to the original studies I cannot suggest whether they would have been judged to be a higher or even lower quality than that suggested here. On a general note, though, this matters because it affects our confidence in the results. If using the observational tool were to suggest the studies were of higher quality than using the Cochrane tool then we would feel more inclined to accept the individual studies' results, and possibly those of the metaanalysis. The authors suggest we read the results with caution due to the poor quality of included studies. This is quite possibly warranted but may not be. If not, then we might be more comfortable concluding there is no increased risk of placing implants in well-controlled diabetics than non-diabetics.

Overall there appeared from their meta-analysis to be no difference in the success rate of implants whether placed in non-diabetics or variously defined 'well-controlled' diabetics. It seems to me that there was a lot of methodological and clinical heterogeneity in the included results both because of the study designs (methodological) and the eligible patients (clinical). I am not convinced, therefore, that it was reasonable to combine the results. However, even if they did, I think at least restricting it to studies of a similar type would have been more appropriate, eg prospective cohort studies alone.

A final relatively minor criticism of this paper is that the authors followed the PRISMA reporting guidance in the body of the paper and the appendix but the abstract lacked key information that PRISMA recommends. ${ }^{3}$ These included the data sources, study eligibility criteria, participants and study appraisal. Given the major problem many non-academics have in accessing full text articles, the abstract may be a key information source and, at least, help in deciding whether to pay for the full article.

In summary, it is currently unclear whether Type I or II diabetes in themselves increase the risk of implant failure and secondly, the degree to which different levels of glycaemic control affects this risk

Dominic Hurst

Department of Adult Oral Health, Barts and The London School of Medicine and Dentistry, Queen Mary University of London, London, UK and Department of Primary Care Health Sciences, University of Oxford, Oxford, UK

1. Box 6.3.a pp 122, Cochrane Handbook for Systematic Reviews of Interventions Higgins JPT, Green S, editors. Chichester, West Sussex: The Cochrane Collaboration and John Wiley \& Sons Ltd; 2009.

2. Sanderson S, Tatt ID, Higgins JP. Tools for assessing quality and susceptibility to bias in observational studies in epidemiology: a systematic review and annotated bibliography. Int / Epidemiol 2007; 36: 666-676. PubMed PMID: 17470488. Epub 2007/05/02. eng.

3. Liberati A, Altman DG, Tetzlaff J, et al. The PRISMA statement for reporting systematic reviews and meta-analyses of studies that evaluate health care interventions: explanation and elaboration. PLoS Med 2009; 6: e1000100. PubMed PMID: 19621070. Pubmed Central PMCID: 2707010. Epub 2009/07/22. eng.

Evidence-Based Dentistry (2014) 15, 102-103. doi:10.1038/sj.ebd.6401057 